\title{
Sex differences in radial maze performance: Influence of rearing environment and room cues
}

\author{
PENNY SEYMOURE, HUI DOU, and JANICE M. JURASKA \\ University of Illinois, Champaign, Illinois
}

\begin{abstract}
There have been conflicting reports on whether or not male rats outperform females in the radial arm maze. Three factors - rearing environment, room cues, and whether all of the maze arms are baited with food-may differentially influence the performance of male and female rats. In the present experiment, littermate male and female hooded rats were reared either in same-sex groups in a complex environment or alone in standard cages for 30 days after weaning. The rats were then trained for 24 days in a partially baited 17-arm radial maze with either a minimum of room cues or a large number of cues. Regardless of rearing condition or number of cues, males made fewer errors (baited and unbaited) than did females, and both sexes reared in the complex environments made fewer errors (baited and unbaited) than did the rats reared alone.
\end{abstract}

The radial maze has been a popular instrument for investigating how male and female rats navigate through space. This maze minimizes errors caused by exploration and/or high activity, usually committed by female rats in other types of mazes (for reviews, see Beatty, 1979; van Haaren, van Hest, \& Heinsbroek, 1990). However, when sex differences have been examined in the radial maze, the results have been inconsistent, although any reported differences in learning have always favored male rats. In this experiment we examined whether rearing environment or extramaze room cues influence the appearance of sex differences in performance in a radial maze with baited and unbaited arms.

Numerous studies have reported that rearing environments affect maze performance. Rats that have been reared with objects and other rats in a complex environment (EC) exhibit superior performance in learning a variety of mazes compared with rats raised alone in standard laboratory cages (IC). These results have been obtained with the Davenport maze (Joseph \& Gallagher, 1980), the Lashley III maze (Greenough, Madden, \& Fleischmann, 1972), the HebbWilliams maze (Brown, 1968; Hymovitch, 1952; Joseph, 1979; Smith, 1972), and the 8-arm (Einon, 1980) and 17arm (Juraska, Henderson, \& Müller, 1984) radial maze.

There is conflicting evidence about whether the rearing environment alters the performance of both sexes on the radial maze. Einon (1980) reported that male rats in EC learned an 8-arm radial maze faster than male rats in IC, while there was no difference in performance between the two groups of female rats. However, in two replications in our laboratory, there were no sex differences in performance in a 17-arm radial maze between littermate pairs of

This study was supported by NSF IBN 9310945 . We thank M. E. Walker and L. Hulbert for their helpful advice on the statistical analysis programs. Correspondence should be addressed to J. M. Juraska, Department of Psychology, University of Illinois, Champaign, IL 61820 (email:jjuraska@s.psych.uiuc.edu). male and female hooded rats reared in the same environment (Juraska et al., 1984). There was no difference between socially housed male and female albino rats on an 8-arm radial maze (van Haaren, Wouters, \& van de Poll, 1987), while a small sex difference in performance on a 17-arm radial maze was reported between male and female hooded rats that had been reared either in the light or the dark (Tees, Midgley, \& Nesbit, 1981).

Another salient factor that may affect maze performance is how food is placed in the maze arms, specifically, whether all the arms or just a subset of the arms contain food at the onset of the daily trial. In the studies reviewed above, all the arms had food. Some rats, especially those reared in $\mathrm{EC}$, developed an adjacent arm strategy to learn to locate food (Einon, 1980; Juraska et al., 1984). One way to break this response algorithm is to put food in a subset of arms. Although the effect of rearing environment has not been examined with this method, sex differences in performance have emerged in some studies. Male rats (Beatty, 1984; Williams, Barnett, \& Meck, 1990) and male mice (Mishima, Higashitani, Teraoka, \& Yoshioka, 1986) made fewer working memory (baited) and reference memory (unbaited) errors than females when only a subset of arms contained food. However, two other studies failed to find a sex difference in rats (Maier \& Pohorecky, 1986; Schwegler, Mueller, Crusio, Szemes, \& Seress, 1993).

Extramaze cues are a factor that may interact with sex and thus amplify performance differences in the radial maze. Unfortunately, the number of extramaze cues in the room has differed between studies from none or few (Juraska et al., 1984; Tees et al., 1981) to numerous (Tees et al., 1981; van Haaren et al., 1987). Poucet, Lucchessi, and ThinusBlanc (1991) have recently suggested that male rats use two types of information, local views and stored maps, in a complementary manner to update the location of arms containing food. Previously, Cheng and Gallistel (Cheng, 1986; Cheng \& Gallistel, 1984; Margules \& Gallistel, 1988) proposed that male rats rely on room geometry rather than 
local featural cues within a testing chamber on the basis that they make more working and reference memory errors when the geometric cues are rotated. Williams, Barnett, and Meck (1990) tested Cheng and Gallistel's premise on a 12-arm radial maze with food in 8 arms. They reported that male rats selectively attend to the geometric aspects of the testing room and ignore featural or landmark cues if the room geometry is unambiguous, but rely on the featural or landmark cues when the room is cylindrical. Female rats used more aspects of the environment - both the shape of the room and the movable objects in the room-as landmarks to establish specific spatial locations. This resulted in females' making more errors while learning the maze.

On the basis of the Williams et al. (1990) experiment, we investigated the conditions under which sex differences may exist in the 17-arm radial maze in the present experiment. We hypothesized that we would find similar performance between males and females in a maze condition without many cues, but in a condition with prominent landmark and geometric cues, males would learn the maze more rapidly because they would not attend to as many cues as would females. So we expected that any potential sex difference in performance would interact with the number of cues available.

In addition to our interest in potential sex differences in performance, we also wanted to know if rats raised in EC, which featured a changing landscape, would utilize cues in the same manner as rats that had been housed in IC and exposed to a retatively static environment. In previous work from our laboratory (Juraska et al., 1984), the rats raised in EC exhibited superior performance in part because they were adept at using an adjacent arm algorithm. In the present experiment, we weakened or removed this advantage by baiting only 11 quasirandomly positioned arms to discourage adjacent arm responding.

\section{METHOD}

\section{Subjects}

The subjects were 26 male and 26 female hooded Long-Evans rats born in the psychology department animal colony. The animals were first-generation descendants from Simonsen Laboratories (Gilroy, CA) stock. Of the 52 animals, 12 littermate sets consisting of 2 males and 2 females were tested in the maze. The additional 2 males and 2 females were used as weight controls.

\section{Housing}

All procedures regarding housing, handling, and maze apparatus were identical to those used by Juraska et al. (1984). At 25 days of age, the animals were weaned and either group housed in a complex environment or placed alone in an isolated cage. In the EC condition, 1 male and 1 female from each litter were housed with 12 other same-sex rats in a large wire-mesh cage $(80 \times 82 \times 90 \mathrm{~cm})$. The cage was half filled with an assortment of metal and plastic toys that were changed on a daily basis. These subjects were also put in a $1.2 \times 1.2 \mathrm{~m}$ box that was filled with a different arrangement of toys for 30 min each day. The remaining 13 male and 13 female rats were individually housed in standard plastic cages $(42 \times 24 \times 20 \mathrm{~cm})$ and handled for $5 \mathrm{~min}$ twice a week. This resulted in the rats in isolated cages receiving approximately as much total handling time as those in EC, who were handled very briefly each day. Previous re- search by Greenough et al. (1972) showed that daily handling of IC rats does not change differences in performance for EC and IC rats in the Lashley III maze. All animals were housed in the same room on a 12:12-h light:dark cycle. All handling and maze procedures occurred during the subject's light phase. Food and water were ad lib until maze training began.

\section{Apparatus}

The central platform ( $86 \mathrm{~cm}$ in diameter) and the 17 radiating arms were elevated $65 \mathrm{~cm}$ above the floor. Each arm was $69 \mathrm{~cm}$ long and $10 \mathrm{~cm}$ wide, with a $3-\mathrm{cm}$ wall along each side. The maze was painted a flat gray. During all the maze trials a metal bookcase for supplies, the experimenter's chair, and a door were located along the north wall of a nearly square room $(3.56 \times 3.76 \mathrm{~m})$. One half of the litters were tested in a low-cue condition with the minimum extramaze cues described above. The remaining litters (high-cue condition) were tested in the same room; however, both landmark and geometric cues were added to the three bare walls. High-contrast posters were placed on two walls (landmark cues) and the shape of the room (geometric cue) was changed by hanging a patterned sheet across one corner.

\section{Procedure}

At the end of 30 days in the housing conditions, the rats were placed on restricted food intake and reduced to $85 \%$ of their body weight. During maze training and testing, their weight was maintained at $85 \%$ of their free-feeding weight by comparing them to the percentage gains in weight on a control rat of comparable sex and rearing environment. All rats were weighed every other day. Rats housed in the EC condition were placed in individual cages at the beginning of each day so that they could be sorted for running and later feeding, then returned to the EC cage at the end of the day. For 4 days, each rat was given 12 min to explore the 17 -arm maze with all the arms baited. At the end of the training period, all the rats were finding and eating the 190-mg pellet placed in a recessed food well at the end of each arm.

The animals were tested on one trial a day for 6 days per week for a total of 24 testing days. During testing, 11 maze arms were baited and Arms 2, 3, 6, 10, 14, and 17 were left unbaited. These arms were picked so that no obvious 1-, 2-, 3-, or 4-adjacent-arm pattern could be discerned. The spatial position of the arms never varied during maze testing. The rats were removed from the maze after successfully locating each food pellet or at the end of $15 \mathrm{~min}$. An experimenter sat near the maze and scored errors for (1) each reentry into a baited arm (working memory error) and (2) each entry into an unbaited arm (reference memory error). An error was counted when the animal was observed placing both front paws into an incorrect $\mathrm{arm}$. The sequence of arms visited and the total number of arms entered were also recorded.

The maze was cleaned between each animal to discourage the use of intramaze cues. To prevent the buildup of food odors within a given arm, each arm was physically rotated counterclockwise by two arm positions at the end of every 4th day. Thus, the spatial position of baited arms was always the same, but the physical position of arms that did or did not contain food was routinely changed.

\section{Statistics}

A four-factor (cue condition, environment, sex, days) analysis of variance (ANOVA) with days as a repeated measure was performed using the SAS statistical package. The repeated factor of days was analyzed in blocks of 2 days or blocks of 8 days. Error scores for incorrect baited and unbaited arm entries, the number of correct choices before the first error and in the first 11 arms entered, and the number of arms visited were analyzed separately over all 24 days. As in Juraska et al. (1984), blocks of 2 days were used to observe overall performance within and between the subjects. To make a more direct comparison with Williams et al. (1990), blocks of 8 days were used to analyze how performance changed between groups during the first, middle, and final days of testing. 


\section{RESULTS}

\section{Sex Differences}

Males outperformed females on all measures except for the number of correct choices before the first error (Table 1). Overall, males made fewer baited (working memory) errors (Figure 1) and unbaited (reference memory) errors (Figure 2) than did females. Males made more correct choices in the first 11 arms and completed the maze by visiting fewer arms than did females over all trials.

When the trials were broken into three blocks of 8 days, the significant male-female differences in baited errors occurred during the first 16 days of testing (Figure 3). Likewise, sex differences for correct choices in the first 11 arms $(p<$ $.007, p<.02)$ and total number of arms to completion $(p<$ $.01, p<.01$ ) were significant only during Days $1-8$ and $9-16$, respectively. The sex difference in unbaited errors faded to nonsignificance after Days 1-8 (Figure 4).

\section{Environmental Housing Differences}

Rats raised in EC had significantly better performance scores than rats raised in IC with the exception of the number of correct choices made before the first error (Table 1). Rats housed in EC had fewer baited errors (Figure 1) and fewer unbaited errors (Figure 2) over the entire 24 days than rats raised in IC housing. Rats raised in EC made more successful choices in the first 11 arms and made fewer arm entries over the 24 days of testing than rats in IC.

Table 1

The Overall Means and Standard Errors for

\begin{tabular}{|c|c|c|c|c|}
\hline & $M$ & manc & Factor & Significance \\
\hline \multicolumn{5}{|c|}{ Baited Arm Errors } \\
\hline Male & 4.69 & 0.19 & Trials & $p<.0001$ \\
\hline Female & 6.80 & 0.23 & Sex & $p<.0009$ \\
\hline EC & 4.45 & 0.20 & Environment & $p<.0001$ \\
\hline IC & 7.04 & 0.22 & & \\
\hline \multicolumn{5}{|c|}{ Unbaited Arm Errors } \\
\hline Male & 6.09 & 0.13 & Trials & $p<.0001$ \\
\hline Female & 6.94 & 0.14 & Sex & $p<.03$ \\
\hline EC & 5.92 & 0.12 & Environment & $p<.004$ \\
\hline IC & 7.11 & 0.15 & & \\
\hline \multicolumn{5}{|c|}{ Number Correct Choices in 1st 11 Arms } \\
\hline Male & 7.50 & 0.06 & Trials & $p<.0001$ \\
\hline Female & 7.09 & 0.06 & Sex & $p<.03$ \\
\hline $\mathrm{EC}$ & 7.58 & 0.05 & Environment & $p<.007$ \\
\hline IC & 7.01 & 0.06 & & \\
\hline \multicolumn{5}{|c|}{ Number Correct Choices Before 1st Error } \\
\hline Male & 2.69 & 0.10 & Trials & $p<.0001$ \\
\hline Female & 2.40 & 0.09 & Sex & n.s. \\
\hline $\mathrm{EC}$ & 2.61 & 0.10 & Environment & n.s. \\
\hline IC & 2.49 & 0.09 & & \\
\hline \multicolumn{5}{|c|}{ Total Arms to Completion } \\
\hline Male & 21.50 & 0.29 & Trials & $p<.0001$ \\
\hline Female & 24.15 & 0.34 & Sex & $p<.004$ \\
\hline $\mathrm{EC}$ & 21.19 & 0.29 & Environment & $p<.0007$ \\
\hline IC & 24.46 & 0.34 & & \\
\hline
\end{tabular}

Note-EC, complex environment; IC, individually caged.

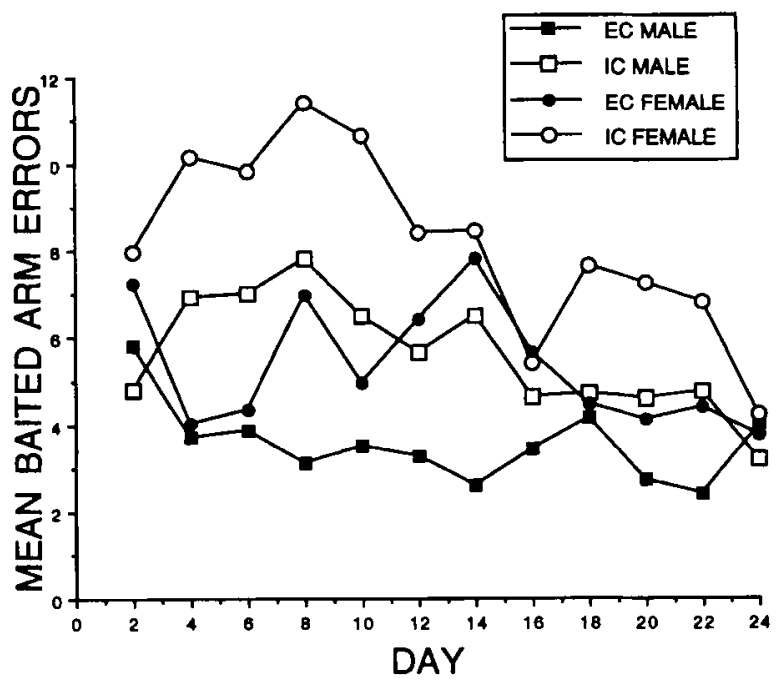

Figure 1. The mean number of baited arm entry (working memory) errors for male and female rats reared in the two housing conditions is shown in 2-day increments. EC, complex environment; IC, individually caged.

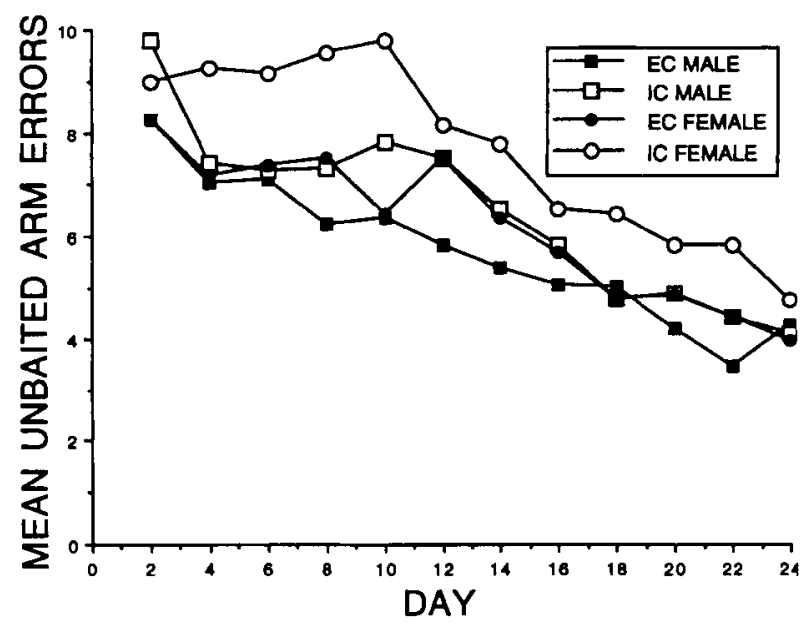

Figure 2. The mean number of unbaited arm entry (reference memory) errors for males and females reared in the two housing conditions is shown in 2-day increments. EC, complex environment; IC, individually caged.

The rats reared in EC maintained a significant advantage over rats reared in IC in errors into baited arms (Figure 3 ) and in the number of arms entered during the first $(p<.0001)$, middle $(p<.03)$, and last $(p<.03) 8$ days of testing. However, the number of errors into unbaited arms (Figure 4) and correct choices in the first $11 \mathrm{arms}$ were significantly different between the groups only for testing Days $1-8(p<.0004)$ and $9-16(p<.006)$.

\section{Cue Condition Differences}

There was no significant overall main effect for the cue condition on any measure. However, the subjects in the 


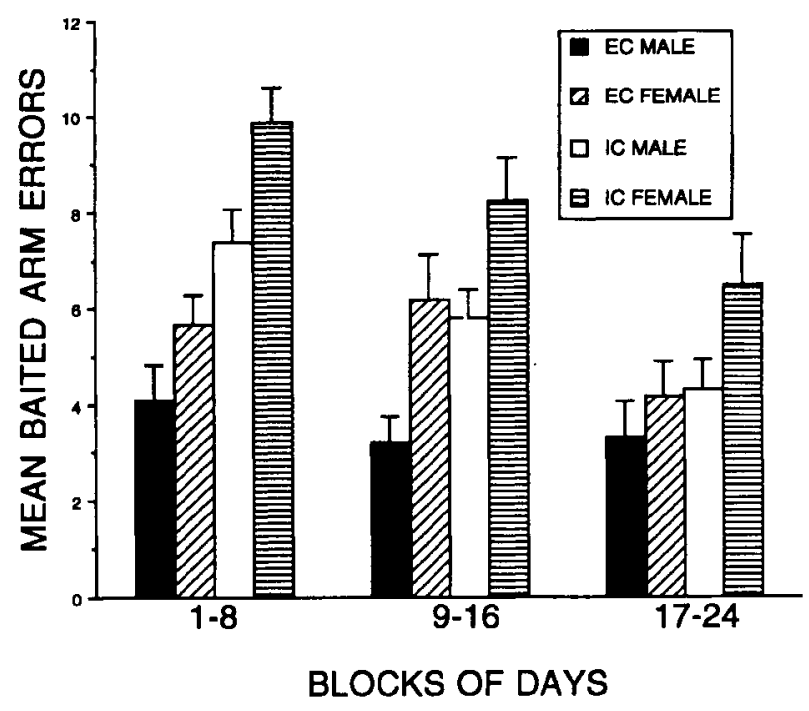

Figure 3. The means and standard errors for baited arm entry (working memory) errors during the first, middle, and last 8 days of maze testing are illustrated for each sex and environment group. Significant differences were found for the main effects of sex on Days 1-8 $(p<.009)$ and 9-16 $(p<.001)$, and environment for Days 1-8 $(p<.0001), 9-16(p<.004)$, and 17-24 $(p<.04)$. EC, complex environment; IC, individually caged.

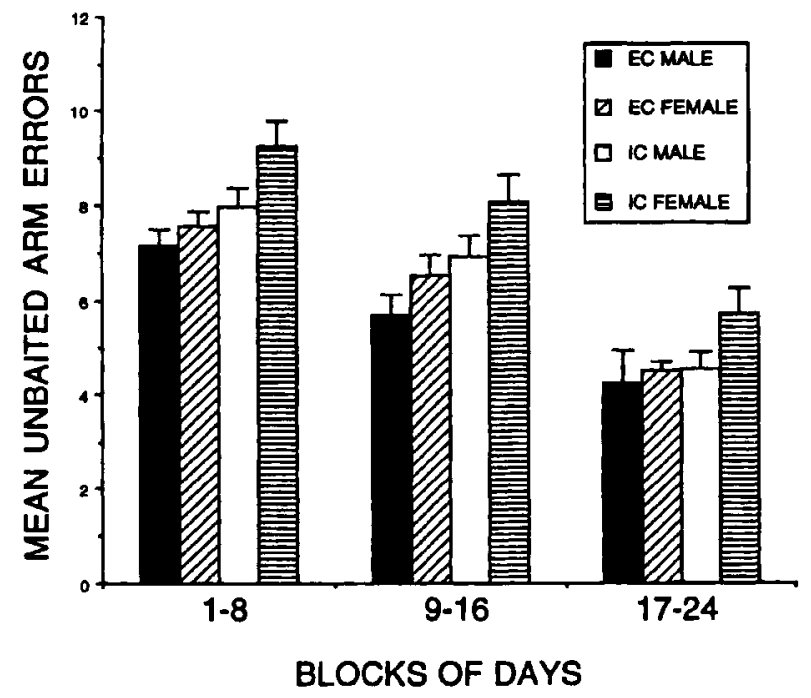

Figure 4. The means and standard errors for unbaited arm entry (reference memory) errors during the first, middle, and last 8 days of maze testing are displayed as sex $\times$ environment groups. Significant differences were found for the main effects of sex on Days 1-8 $(p<.04)$ and environment for Days 1-8 $(p<.004)$ and 9-16 $(p<.007)$. EC, complex environment; IC, individually caged.

high-cue condition made significantly fewer baited entry errors $(p<.008)$ and entered fewer total arms $(p<.04)$ during the last 8 days of testing than did the subjects in the low-cue condition.

\section{Interactions}

There were no significant interactions between sex, housing environment, and cue condition on any of the measures.

\section{Days Factor}

The repeated days factor was significant for every performance measure, including the number of correct choices made before the first error $(p<.0001)$. This indicates that performance on every measure improved with repeated training.

\section{DISCUSSION}

In the absence of any significant interactions between sex and rearing environment, our results clearly indicated that within each environment, male rats were more efficient at learning the baited-unbaited 17-arm radial maze than were female rats. The sex difference appeared regardless of the shape of the room or the number of objects that could serve as extramaze cues. This was especially evident in the error scores and in the number of arms entered during the early maze trials. Furthermore, we found that rats that had been raised in a relatively complex environment with varying objects were better at learning the maze than rats raised in isolated cages, whether or not room cues were abundant.

We did not find an interaction between sex and the number of cues in the room, as we hypothesized given the work of Williams et al. (1990). In the Williams et al. experiment, male rats appeared unperturbed by alterations in the arrangement of landmark cues, but their performance was seriously hampered when the room geometry was changed. In the present experiment, there was no difference between the overall performance of males who were tested with either numerous or few room cues. We had expected that females would be slower than males to learn the maze in the presence of many cues because females might be using the landmark and geometric cues to solve the maze. However, both males and females with many cues may have eventually benefited from the additional cues because they showed a significant decrease in working memory errors during the last third of the maze trials. Unlike Williams et al., we did not test each animal in both cue conditions, so we do not know if experience with learning the maze under one cue condition could impair or improve performance with a different type of cue condition.

Another important distinction between these two studies is that all of the animals used by Williams et al. (1990) had undergone some form of surgical and hormonal intervention, while all of our animals were hormonally intact. Some of their subjects were deprived of normal gonadal hormones shortly after birth, and all of their subjects were gonadectomized by 45 days of age. Even their control animals were lacking gonadal steroids in a period of time that overlaps puberty. Moveover, these control animals had been without any gonadal hormones for approximately 60 to 80 days prior to the completion of maze testing. Thus, comparisons to intact animals may not be appropri- 
ate and we can only tenuously predict that the behaviors of experimental animals will be the same in animals that have had a normal hormonal milieu throughout life.

In the present experiment and in earlier ones, male rats (Beatty, 1984; Williams et al., 1990) and male mice (Mishima et al., 1986) were faster at learning the radial maze when the majority of arms were baited with food and when they could not easily use an alternate arm strategy. In each study, the magnitude of error difference between males and females was greatest in the number of baited errors (the working memory task) where the subject must keep track of the arms it has visited that day. As in our study, Williams et al. (1990) reported that their male controls made fewer baited arm (working memory) errors than did the female controls in the beginning days of the training sessions, which resulted in a different learning curve for each sex. One possible explanation for the lack of sex differences in performance in the 8-arm radial maze when only 4 of the arms have been baited (Maier \& Pohorecky, 1986; Schwegler et al., 1993) is that the working memory load is greatly reduced in comparison with a radial maze that requires the subject to continually update entries into 8 or 11 arms.

It is not readily apparent why male rats demonstrate better working memory and, to a lesser degree, reference memory skills than do female rats in larger or more complex radial arm mazes, such as the baited-unbaited version of the 17-arm radial maze. These sex differences occur regardless of the level of the postweaning environmental stimulation or the number of cues in the room. In the radial maze, working memory is impaired when either the hippocampal system (Becker, Walker, \& Olton, 1980) or the prefrontal cortex (Kesner, 1989; Kolb, Sutherland, \& Whishaw, 1983) is lesioned, while reference or retrospective memory is impaired after damage to the parietal cortex (Kametani \& Kesner, 1989). Sex differences in cellular anatomy have been described in several regions of the hippocampus (Juraska, 1990; Madeira, Sousa, \& Paula-Barbosa, 1991 ) and the prefrontal cortex (Kolb \& Stewart, 1991) of the rat. How these sex differences in behavior are related to the complicated pattern of sex differences in the structure of the rat hippocampus and cerebral cortex remains to be elucidated.

\section{REFERENCES}

BEAtTY, W. W. (1979). Gonadal hormones and sex differences in nonreproductive behaviors in rodents: Organizational and activational influences. Hormones \& Behavior, 12, 112-163.

BeatTy, W. W. (1984). Hormonal organization of sex differences in play fighting and spatial behavior. Progress in Brain Research, 61, 315-330.

Becker, J. T., Walker, J. A., \& Olton, D. S. (1980). Neuroanatomical bases of spatial memory. Brain Research, 200, 307-320.

Brown, R. T. (1968). Early experience and problem-solving ability. Journal of Comparative \& Physiological Psychology, 65, 433-440.

CHENG, K. (1986). A purely geometric module in the rat's spatial representation. Cognition, 23, 149-178.

Cheng, K., \& Gallistel, C. R. (1984). Testing the geometric power of an animal's spatial representation. In H. L. Roitblat, T. G. Bever, \& H. S. Terrace (Eds.), Animal cognition (pp. 409-423). Hillsdale, NJ: Erlbaum.
EINON, D. (1980). Spatial memory and response strategies in rats: Age, sex and rearing differences in performance. Quarterly Journal of Experimental Psychology, 32, 473-489.

Greenough, W. T., Madden, T. C., \& Fleischmann, T. B. (1972). Effects of isolation, daily handling, and enriched rearing on maze learning. Psychonomic Science, 27, 279-280.

Hymovitch, B. (1952). The effects of experimental variations on problem solving in the rat. Journal of Comparative \& Physiological Psychology, 45, 313-321.

JOSEPH, R. (1979). Effects of rearing and sex on maze learning and competitive exploration in rats. Journal of Psychology, 101, 37-43.

JosePH, R., \& GallaGHer, R. E. (1980). Gender and early environmental influences on activity, overresponsiveness, and exploration. Developmental Psychobiology, 13, 527-544.

JuRASKA, J. M. (1990). The structure of the rat cerebral cortex: Effects of gender and environment. In B. Kolb \& R. C. Tees (Eds.), The cerebral cortex of the rat (pp. 483-505). Cambridge, MA: MIT Press.

Juraska, J. M., Henderson, C., \& Müller, J. (1984). Differential rearing experience, gender, and radial maze performance. Developmental Psychobiology, 17, 209-215.

Kametani, H., \& Kesner, R. P. (1989). Retrospective and prospective coding of information: Dissociation of parietal cortex and hippocampal formation. Behavioral Neuroscience, 103, 84-89.

KESNER, R. P. (1989). Retrospective and prospective coding of information: Role of the medial prefrontal cortex. Experimental Brain Research, 74, 163-167.

Kolb, B., \& STEWART, J. (1991). Sex-related differences in dendritic branching of cells in the prefrontal cortex of rats. Journal of Neumendocrinology, 3, 95-99.

Kolb, B., Sutherland, R. J., \& WhishaW, I. Q. (1983). A comparison of the contributions of the frontal and parietal association cortex to spatial localization in rats. Behavioral Neuroscience, 97, 13-27.

Madeira, M. D., Sousa, N., \& Paula-Barzosa, M. M. (1991). Sexual dimorphism in the mossy fiber synapses of the rat hippocampus. $E x-$ perimental Brain Research, 87, 537-545.

MaIER, D. M., \& POHORECKy, L. A. (1986). The effect of ethanol and sex on radial arm maze performance in rats. Pharmacology, Biochemistry \& Behavior, 25, 703-709.

Margules, J., \& Gallistel, C. R. (1988). Heading in the rat: Determination by environmental shape. Animal Learning \& Behavior, 16, 404-410.

Mishima, N., Higashitani, F., Teraoka, K., \& Yoshioka, R. (1986). Sex differences in appetitive learning in mice. Physiology \& Behavior. 37, 263-268.

Poucet, B., Lucchessi, H., \& Thinus-Blanc, C. (1991). What information is used by rats to update choices in the radial-arm maze? $B e$ havioural Processes, 25, 15-26.

Schwegler, H., Mueller, G. G., Crusio, W. E., Szemes, L., \& SerESs, L. (1993). Hippocampal morphology and spatially related behavior in Long-Evans and CFY rats. Hippocampus, 3, 1-8.

Sмітн, H. V. (1972). Effects of environmental enrichment on open-field activity and Hebb-Williams problem solving in rats. Journal of Comparative \& Physiological Psychology, 80, 163-168.

TEes, R. C., Midgley, G., \& NesBit, J. C. (1981). The effect of early visual experience on spatial maze learning in rats. Developmental Psychobiology, 14, 425-438.

van Haaren, F., van Hest, A., \& Heinsbroek, R. P. W. (1990). Behavioral differences between male and female rats: Effects of gonadal hormones on learning and memory. Neuroscience \& Biobehavioral Reviews, 14, 23-33.

van Haaren, F., Wouters, M., \& van de Poll, N. E. (1987). Absence of behavioral differences between male and female rats in different radial-maze procedures. Physiology \& Behavior, 39, 409-412.

Williams, C. L, BarnetT, A. M., \& Meck, W. H. (1990). Organizational effects of early gonadal secretions on sexual differentiation in spatial memory. Behavioral Neuroscience, 104, 84-97.

(Manuscript received January 12, 1995; revision accepted for publication December 2, 1995.) 